ARQGA/1143

\title{
APRESENTAÇÃO CLÍNICA DE DOENÇA CELÍACA EM CRIANÇAS DURANTE DOIS PERÍODOS, EM SERVIÇO UNIVERSITÁRIO ESPECIALIZADO
}

\author{
Lívia Carvalho GALVÃO ${ }^{1}$, José Mário Martins BRANDÃO ${ }^{3}$, \\ Maria Inez Machado FERNANDES ${ }^{1}$ e Antonio Dorival CAMPOS ${ }^{2}$
}

RESUMO - Racional - Estudos demonstram mudança na forma de apresentação clínica da doença celíaca, com aumento da ocorrência de formas atípicas tanto em crianças, como em adultos. Objetivo - Verificar este fato em crianças atendidas em serviço universitário especializado (Hospital das Clínicas da Faculdade de Medicina de Ribeirão Preto, SP.). Métodos - Foram estudados pacientes celíacos em dois períodos: de janeiro de 1978 a dezembro de 1987 (grupo $1=$ G1) e janeiro de 1988 a dezembro de 1997 (grupo 2 = G2). Foram analisados: tempo de doença, idade ao diagnóstico, estado nutricional e formas clínicas. Considerou-se forma típica se o paciente tinha pelo menos dois dos seguintes sinais/sintomas: diarréia, distensão abdominal, perda de peso, associados ou não a outros. A análise estatística foi realizada pelos testes exato de Fisher e de Wilcoxon. Resultados - Mediana de idade ao diagnóstico: 23 meses (G1) e 21 (G2). Intervalo entre início de sintomas e diagnóstico: 13 meses (G1) e 11 (G2). Estado nutricional (\%) Gomez: G1:eutrofia =23,8; DI = 47,61; DII = 23,8 e DIII = 4,76. $\mathrm{G} 2$ : eutrofia $=20,68, \mathrm{DI}=48,27, \mathrm{DII}=27,58 \mathrm{e} \mathrm{DIII}=3,44$. Waterloo: $\mathrm{G} 1$ : eutrofia $=23,8$, emaciação $=14,28$ baixa estatura $=28,57 \mathrm{e}$ crônico $=33,33$. G2: eutrofia $=20,68$, emaciação $=13,79$, baixa estatura $=34,48$ e crônico $=31,03$. Formas clínicas $(\%)$ - G1: típica 57,14 e atípica 42,85. G2: típica 55,18 e atípica 44,82. Nenhum dado foi estatisticamente diferente nos dois grupos. Conclusões - No estudo não se encontrou mudança nas formas de apresentação e demais características clínicas da doença celíaca. Cogita-se que essa hipótese poderia ser decorrente de características populacionais, de diferenças na abordagem nos serviços de saúde ou de diferentes períodos de observação, em comparação com os demais estudos.

DESCRITORES - Doença celíaca. Diarréia. Desnutrição protéico-energética.

\section{INTRODUÇÃO}

A doença celíaca (DC) é uma das principais causas de diarréia crônica em crianças. A forma típica é expressa clinicamente por síndrome de má absorção intestinal, com repercussões sobre o estado nutricional dos pacientes. $\mathrm{O}$ aspecto anatomopatológico de mucosa de intestino delgado caracteriza-se por atrofia vilositária, hiperplasia de criptas, aumento dos linfócitos intraepiteliais e infiltração da lâmina própria da mucosa por células inflamatórias $^{(1)}$. Sua etiologia está relacionada a fatores ambientais (ingestão de glúten), imunológicos (alterações da imunidade celular e humoral) e genéticos ${ }^{(12,24,25)}$.

Atualmente, a teoria mais aceita para a patogênese da DC baseia-se na associação entre a ingestão de glúten e a predisposição genética. Há indícios de que uma região $\mathrm{N}$-terminal da gliadina seja responsável por desencadear resposta imunológica mediada por linfócitos T que, por sua vez, seria responsável pelos danos à mucosa intestinal. Sabe-se que os alelos DQA1 0501 e DQB1 0201 estão envolvidos neste processo ${ }^{(8,24,29)}$.
A DC é predominante em indivíduos caucasianos; os nãocaucasianos representam uma minoria dos casos. Quanto ao sexo, sabe-se que a relação feminino/masculino oscila entre 1,4 e $2^{(3,8)}$. A prevalência varia segundo a região estudada, mas também de acordo com a metodologia utilizada nos estudos. Na Europa, oscila entre 1:150 e 1:300 ${ }^{(1,8)}$. Em estudos recentes envolvendo doadores de sangue, detectou-se freqüência de 1:250 nos $\mathrm{EUA}^{(1)}$ e no Brasil, freqüências de 1:681 (Brasília) ${ }^{(11)}$ e 1:273 (Ribeirão Preto) ${ }^{(18)}$.

Conforme critérios diagnósticos estabelecidos pela Sociedade Européia de Gastroenterologia Pediátrica e Nutrição (ESPGAN), em $1970^{(19)}$, a DC é caracterizada como:

a) sintomas e sinais de má absorção intestinal;

b) mucosa jejunal de aspecto celíaco, com vilosidades achatadas, hiperplasia de criptas e infiltrado inflamatório linfoplasmocitário de submucosa;

c) remissão clínica e histológica quando submetida a dieta sem glúten;

d) recurrência clínica e histológica após a reintrodução do glúten.

Hospital das Clínicas da Faculdade de Medicina de Ribeirão Preto da Universidade de São Paulo - FMRP-USP, Ribeirão Preto, SP.

${ }^{1}$ Departamento de Puericultura e Pediatria; ${ }^{2}$ Departamento de Medicina Social FMRP-USP; ${ }^{3}$ Bolsa de Iniciação Científica do programa PIBIC da FMRP-CNPq.

Endereço para correspondência: Dra. Lívia Carvalho Galvão - Departamento de Puericultura e Pediatria - Hospital das Clínicas - $7^{\circ}$ andar - Av. Bandeirantes, 3900 - $14045-900$

- Ribeirão Preto, SP. E-mail: lcgalvao@fmrp.usp.br 
Esses critérios foram revistos em 1990, quando se estabeleceu que a provocação e a terceira biopsia seriam dispensadas nos casos em que os demais critérios fossem positivos e a criança fosse diagnosticada em idade acima de 2 anos.

A detecção de anticorpos como auxiliar diagnóstico veio reforçar tais modificações ${ }^{(10)}$. O anticorpo anti-reticulina no soro dos pacientes foi o primeiro a ser descrito e é determinado por imunofluorescência. A seguir, foi introduzido o anticorpo anti-gliadina que, embora também não apresente especificidade ideal, tem as vantagens, com relação ao anterior, de ser realizado por técnica de ELISA e poder detectar IgG, o que é útil em pacientes com déficit de IgA. Atualmente, a determinação de anticorpos anti-transglutaminase (técnica de ELISA) e o anticorpo anti-endomísio (imunofluorescência) são os mais utilizados, pois apresentam alta sensibilidade e especificidade. Além de auxiliar no diagnóstico da doença nos casos suspeitos, esses anticorpos são muito úteis na detecção de casos atípicos e/ou assintomáticos, bem como em estudos populacionais. Sabendo-se que se tornam negativos com a dieta sem glúten, também são de grande valia no acompanhamento clínico dos pacientes em tratamento ${ }^{(14,22)}$. No entanto, ainda não há dados suficientes para que esses exames substituam a biopsia intestinal.

$\mathrm{Na}$ forma típica ou clássica da DC, os sintomas mais comuns são diarréia crônica, perda de peso, distensão abdominal, apatia, anorexia e palidez. A forma atípica pode apresentar-se de várias formas, como baixa estatura, anemia resistente a tratamento, dor abdominal, osteoporose. Na forma silenciosa a doença existe, há alterações de anticorpos e de histologia de mucosa intestinal, sem manifestações clínicas. Na forma latente a doença só se manifesta por alterações de anticorpos $^{(4,17,19,20,28)}$.

Estudos recentes têm demonstrado modificações na apresentação clinica das distintas formas de DC em crianças e adultos, com aumento das formas atípicas $^{(23,28)}$. Apenas um estudo brasileiro aborda tal assunto ${ }^{(22)}$.

O objetivo deste estudo foi verificar se houve mudança nos aspectos clínicos de apresentação de crianças portadoras de DC, no decorrer de 20 anos de estudo, no Serviço de Gastroenterologia Pediátrica do Hospital das Clínicas da Faculdade de Medicina de Ribeirão Preto.

\section{CASUÍTICA E MÉTODOS}

Os dados foram obtidos dos prontuários médicos de pacientes celíacos atendidos no Serviço de Gastroenterologia Pediátrica do Departamento de Puericultura e Pediatria do Hospital das Clínicas da Faculdade de Medicina de Ribeirão Preto da Universidade de São Paulo, Ribeirão Preto, SP. O estudo foi aprovado pelo Comitê de Ética em Pesquisa dessa instituição, processo HCRP n ${ }^{\circ} 7073 / 2001$.

Estudaram-se todos os pacientes celíacos atendidos nesse serviço no período de janeiro de 1978 a dezembro de 1997, sendo definidos como celíacos os que apresentassem pelo menos quatro dos seguintes critérios:

1. quadro clínico compatível com DC

2. primeira biopsia intestinal compatível com DC

3. resposta clínica à retirada do glúten da dieta

4. segunda biopsia normal após dieta

5. recaída histológica após provocação com glúten.

Após inclusão no estudo, os pacientes foram divididos em dois grupos, segundo o período do primeiro atendimento: grupo 1: pacientes atendidos entre janeiro de 1978 e dezembro de 1987, e grupo 2: atendimento entre janeiro de 1988 e dezembro de 1997.
Compararam-se os seguintes aspectos:

Quadro clínico - os pacientes foram alocados em dois subgrupos: forma típica e forma atípica. Foram considerados portadores de forma típica aqueles que referiram pelo menos dois dos seguintes sinais/ sintomas: diarréia, perda de peso e distensão abdominal, associados ou não a outros ${ }^{(3,6,27)}$. Os demais foram alocados na forma atípica. Foi registrada também a idade dos pacientes por ocasião do diagnóstico.

Estado nutricional - foi avaliado no momento do diagnóstico. Utilizou-se os critérios de GOMEZ ${ }^{(13)}$ e WATERLOO ${ }^{(30)}$, tendo como referência a curva de crescimento do National Center for Health Statistics (NCHS) ${ }^{(13,21,29)}$.

Tempo de doença - considerado o tempo decorrido entre o início dos sintomas e o diagnóstico da doença celíaca.

Foram analisados os dados de 72 pacientes, dos quais 22 foram excluídos do estudo por não preencherem os critérios de inclusão estabelecidos. Os 50 pacientes restantes foram divididos em grupos 1 e 2, conforme os critérios já definidos. O grupo 1 foi composto por 21 e o grupo 2 por 29 pacientes.

No grupo 1 observaram-se 10 pacientes do sexo feminino (47,62\%) e 11 do masculino (52,38\%). No grupo 2, 16 eram do sexo feminino $(55,16 \%)$ e 13 do masculino $(44,89 \%)$.

Análise estatística - foram utilizados os testes de Wilcoxon para amostras independentes e o de Fisher para os parâmetros de forma clínica e estado nutricional ${ }^{(9,15)}$.

\section{RESULTADOS}

Os dados de idade e tempo de diagnóstico dos pacientes (medianas e valores mínimo e máximo) encontram-se na Tabela 1 . Não houve diferença estatisticamente significativa entre os dois grupos tanto em relação à idade do paciente ao diagnóstico $(P=0,41)$, quanto ao intervalo entre início dos sintomas e o diagnóstico $(P=0,14)$.

TABELA1 - Idade dos pacientes ao diagnóstico e intervalo entre o início dos sintomas e diagnóstico (mediana e valores mínimo e máximo)

\begin{tabular}{lll}
\hline & Idade (meses)* & Intervalo (meses)** \\
\hline Grupo 1 & $34,9(14$ e 117 & $24,9(1$ a 106$)$ \\
Grupo 2 & $32,5(11$ e 140$)$ & $18,3(2$ a 110$)$ \\
\hline
\end{tabular}

$* P=0,14$

A avaliação do estado nutricional dos pacientes foi realizada segundo os critérios de GOMEZ ${ }^{(13)}$ e de WATERLOO ${ }^{(30)}$. Conforme se observa nas Figuras 1, 2, 3, não foram encontradas diferenças estatisticamente significativas entre os dois grupos, em nenhum dos parâmetros de cada critério de avaliação.

De acordo com critérios clínicos já estabelecidos na metodologia, foi estudada a freqüência das formas clínicas típica e atípica da doença celíaca nos dois grupos, não havendo diferença estatisticamente significativa, utilizando-se o teste exato de Fisher $(P=0,56)$.

\section{DISCUSSÃO}

Existem poucos dados relativos aos aspectos clínicos da DC no Brasil. Além disto, a maioria destes se referem a casos estudados na década de 70 ou início de 80 , quando as formas atípicas da DC eram 
pouco conhecidas. Os critérios diagnósticos variaram bastante nestes estudos, desde os não explicitados no estudo ${ }^{(16,22)}$ até o preenchimento dos critérios iniciais da ESPGAN ${ }^{(10)}$, o que poderia tornar as comparações menos fidedignas.

Os critérios utilizados para definir formas clínicas da doença não estão bem estabelecidos na literatura. A maioria dos autores considera forma típica ou clássica da doença aquela que apresenta sinais ou sintomas de má absorção intestinal, tais como diarréia, distensão abdominal, perda de peso e desnutrição protéico-energética ${ }^{(5,27,29)}$. No presente estudo estabeleceu-se como forma clássica a presença de dois ou mais destes achados. Quando se compara a proporção de formas atípicas encontrada

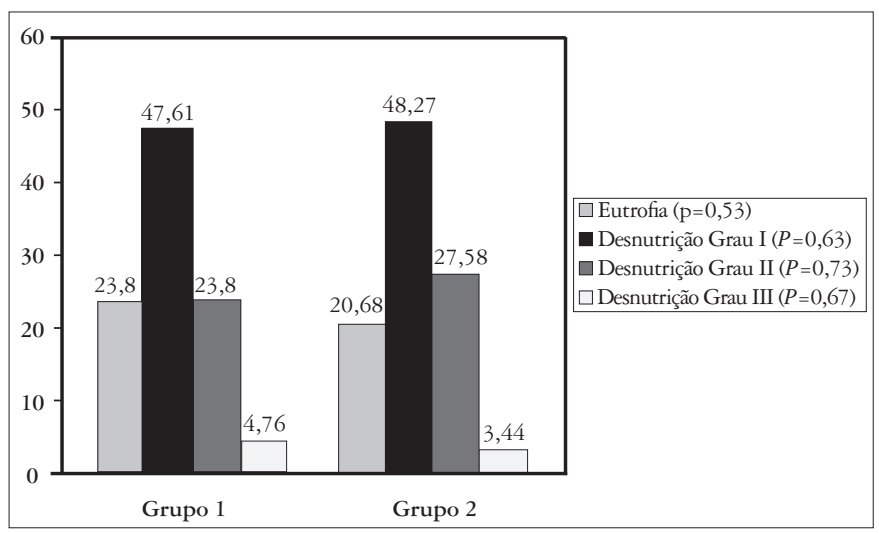

FIGURA 1 - Avaliação do estado nutricional dos pacientes, segundo critério de Gomez

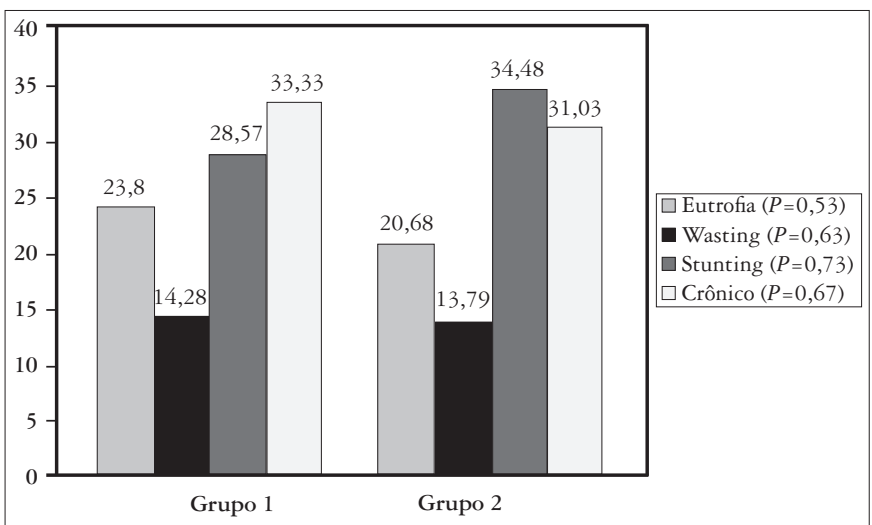

FIGURA 2 - Avaliação do estado nutricional dos pacientes, segundo critério de Waterloo

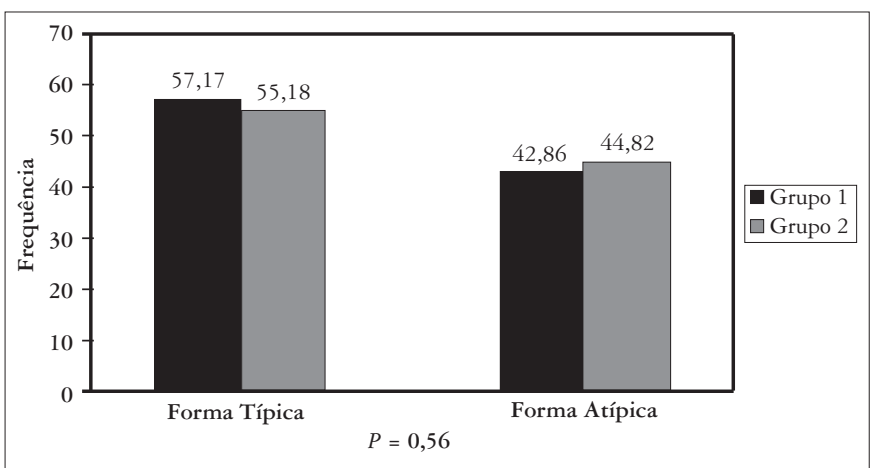

FIGURA 3 - Formas de apresentação clínica da DC nos Grupos 1 e 2 com aquela de dois estudos brasileiros ${ }^{(3,25)}$, observa-se que no serviço em estudo a proporção de formas atípicas da doença em ambos os tempos de observação $(42.9 \%$ e $44.8 \%)$, foi bastante superior àquelas dos outros dois estudos ( $22 \%$ e $16.8 \%$, respectivamente). Este fato provavelmente se deve aos diferentes critérios utilizados. SDEPANIAN et al. ${ }^{(25)}$ estabelecem como critério de definição das formas apenas a presença ou ausência de diarréia, enquanto BARBIERI et al. ${ }^{(3)}$, não explicitam estes critérios.

Decidiu-se, ainda, estudar algumas características que poderiam estar relacionadas às formas clínicas, como idade de aparecimento de sintomas em decorrência do retardo do diagnóstico, como o intervalo entre diagnóstico e início dos sintomas e o estado nutricional das crianças.

Em relação à idade dos pacientes ao diagnóstico, as medianas encontradas para os grupos 1 e 2 ( 23 e 21 meses, respectivamente) não são diferentes da maioria dos estudos nacionais. No I Inquérito Nacional Brasileiro sobre Doença Celíaca ${ }^{(3)}$, cerca de $50 \%$ dos pacientes foram diagnosticados no segundo ano de vida. PENNA et al. ${ }^{(22)}$ e SDEPANIAN et al. ${ }^{(25)}$ diagnosticaram a maior percentagem dos seus casos nos dois primeiros anos. KODA e BARBIERI ${ }^{(16)}$, em 1983, encontram mediana de 12 meses ao diagnóstico, mais precoce que a do presente trabalho, mas não foram definidos os critérios diagnósticos. Como se sabe, em nosso meio as enteropatias de outras etiologias podem se apresentar de forma semelhante à $\mathrm{DC}$, não se pode ter certeza se nestes casos a DC foi excluída posteriormente. GALVÃO et al. ${ }^{(10)}$, em 1992, diagnosticaram casos mais tardiamente (apenas 20\% nos dois primeiros anos de idade).

As medianas do intervalo entre início dos sintomas e diagnóstico foram de 13 e 11 meses para os grupos 1 e 2, respectivamente. No I Inquérito Nacional ...(3), 78\% dos casos foram diagnosticados em intervalos de 1 a 24 meses, ficando a maioria (58\%), entre 1 e 12 meses. Nos demais estudos nacionais, não se dispõem de dados. A ausência de diferenças nos dois períodos, neste estudo, sugere que o encaminhamento dos pacientes ao serviço especializado não sofreu modificações importantes neste tempo.

Com relação ao estado nutricional dos pacientes, encontrou-se menos comprometimento com relação aos demais estudos, nos quais aproximadamente metade das crianças se encontrava em desnutrição de II grau, segundo critério de GOMEZ ${ }^{(13)}$. A maior precocidade de diagnóstico justificaria este achado, mas isto não ocorreu. Assim, talvez as características gerais da população em questão, com menor incidência de agravos nutricionais primários, poderia ser a responsável por estas diferenças. Esta hipótese é, no entanto, especulativa, pois não se tem estudos comprovando ou não este fato. No estudo de SDEPANIAN et al. ${ }^{(25)}$, cuja população se assemelharia mais à aqui estudada, este dado não foi avaliado. Novamente este parâmetro não foi diferente nos dois períodos, o que poderia reforçar a hipótese de condições de assistência à saúde semelhantes em todo o tempo de estudo.

No presente trabalho, a forma clínica predominante foi a forma típica, sem diferenças estatisticamente significantes nos dois períodos de observação. É sabido que há relação entre a idade do aparecimento e formas clínicas da doença. Nas formas típicas, os sintomas geralmente surgem antes dos 2 anos de idade, ao contrário das formas atípicas, mais comuns em crianças maiores e adultos ${ }^{(2,5,7,26)}$. A ausência de diferenças na idade de início dos sintomas vem corroborar a semelhança das formas nos períodos de estudo. 
Três aspectos podem estar ligados às modificações nestas formas: características do serviço (condições de diagnosticar formas atípicas), o encaminhamento de pacientes a serviço especializado e as características populacionais.

Investigações internacionais têm demonstrado mudanças no padrão de apresentação da $\mathrm{DC}$, com predomínio das formas atípicas nos últimos $\operatorname{anos}^{(2,6,7,10)}$. Há, no entanto, controvérsia neste sentido. BOTTARO et al. ${ }^{(5)}$, em estudo feito na Sicília, comparando dois períodos de 1984 a 1989 e 1987 a 1989, não demonstraram diferenças significativas. Esses mesmos autores, em estudo multicêntrico na Itália ${ }^{(6)}$, obtiveram esta diferença na comparação anual do período de 1990 a 1994. Diferenças nas características dos serviços podem explicar as diferenças observadas entre a maioria desses estudos e o presente trabalho, pois em locais onde os problemas básicos de saúde são, na sua maioria, solucionados, as doenças menos comuns são mais conhecidas e detectadas mais eficazmente. Alguns desses estudos relacionam essas mudanças ao surgimento dos anticorpos na investigação clínica da doença, facilitando o diagnóstico das formas atípicas ${ }^{(7)}$. Nos pacientes do Serviço de Gastroenterologia Pediátrica do Hospital das Clínicas da Faculdade de Medicina de Ribeirão Preto, os anticorpos passaram a ser utilizados após o período de observação deste estudo, não tendo sido, portanto, fator de influência nos resultados desta série. As diferenças populacionais também poderiam explicar o fato, mas os estudos populacionais no Brasil, embora escassos, demonstram freqüência de DC na população semelhante aos países europeus ${ }^{(11,18)}$

Apenas um estudo, recente, aborda a evolução das formas clínicas no Brasil ${ }^{(25)}$. Observando pacientes da Associação de
Celíacos, SDEPANIAN et al. ${ }^{(25)}$, em 2001, encontraram diferenças significativas na distribuição das formas em períodos de 5 anos ou mais, anteriores ao estudo. Comparando-se tal estudo com este, não parece provável que as diferenças de serviço ou de população possam ser responsáveis por estas diferenças, visto tratar-se de populações, na sua grande maioria, do mesmo Estado, acompanhadas em serviço universitário. Além disto, a similaridade dos dados de tempo entre sintomas e diagnóstico e estado nutricional nos dois estudos vem reforçar a hipótese de que não parece terem ocorrido diferenças relativas aos aspectos acima citados entre os dois serviços de saúde. Os resultados distintos poderiam ser devido às diferenças nos períodos de observação, mais recentes no estudo de SDEPANIAN et al. ${ }^{(25)}$, quando os anticorpos passaram a ser mais amplamente utilizados no Brasil. No presente estudo, optou-se por analisar períodos mais longínquos porque haveria mais tempo de realizar a biopsia de controle desses pacientes, critério de inclusão dos mesmos. Apesar de se ter como conduta preconizada realizar a biopsia 2 anos após a exclusão de glúten da alimentação, muitas vezes não aderir à dieta é fator de prolongamento desse tempo.

Há que se destacar o tamanho da população em estudo e sua influência nos resultados obtidos. Foi observado que o número de formas atípicas foi maior no último período e talvez, se a amostra fosse maior, esta diferença fosse significativa.

Dessa forma, pode-se concluir que no Serviço aqui estudado não houve diferenças em relação à forma de apresentação clínica da DC, bem como na idade de início de sintomas, tempo entre sintomas e diagnóstico e estado nutricional dos pacientes.

Galvão LC, Brandão JMM, Fernandes MIM, Campos AD. Clinical presentation of children with celiac disease attended at a Brazilian specialized university service, over two periods of time. Arq Gastroenterol 2004;41(4):234-8 .

ABSTRACT - Background - Several studies have demonstrated changes in the forms of clinical presentation of celiac disease in children and adults, with an increased occurrence of atypical forms. Aim - To determine this fact in Clinical Hospital, Ribeirão Preto, SP, Brazil. Methods - Celiac patients were studied over two different periods of time, from January 1978 to December 1987 (group 1 = G1) and from January 1988 to December 1997 (group $2=$ G2). Time of disease, age at diagnosis and nutritional status and clinical forms were investigated. The typical form was considered to be present if the patient had at least two of the following signs/symptoms: diarrhea, abdominal distention, and weight loss, associated or not with others. Results - Clinical forms (\%) - G1: typical 57.14 and atypical 42.85. G2: typical 55.18 and atypical 44.82. Median age at diagnosis: 23 months (G1) and 21 months (G2). Interval between the onset of symptoms and the diagnosis: 13 months (G1) and 11 months (G2). Gomez nutritional status G1: Eutrophy $=23.8$, DI $=47.61$, DII $=23.8$ and DIII $=4.76$. G2: Eutrophy $=20.68, \mathrm{DI}=48.27, \mathrm{DII}=27.58$ and DIII $=3.44$. Waterloo G1: Eutrophy $=23.8$, Wasting $=14.28$, Stunting $=28.57$ and Chronic $=33.33$. G2: Eutrophy $=20.68$, Wasting $=13.79$, Stunting $=34.48$ and Chronic $=31.03$. No statistically significant differences occurred between the groups for any of these parameters. Conclusions - No change in the forms of presentation or remaining clinical characteristics of celiac disease occurred in our service. This may be due to the population characteristics or to differences among health services, or to different periods of observation.

HEADINGS - Celiac disease. Diarrhea. Protein-energy malnutrition.

\section{REFERÊNCIAS BIBLIOGRÁFICAS}

1. American Gastroenterological Association Medical Position Statement. Celiac sprue Gastroenterology 2001;120:1522-5.

2. Barbato M, Miglietta MR, Viola F, Iulianella VR, Frediani T, Lucarelli S, Tozzi AE, Cardi E. Impact of modification of diagnostic techniques and criteria on the presentation of celiac disease in the last 16 years. Observation in Rome. Minerva Pediatr 1996;48:359-63.

3. Barbieri D, Koda YKL, Rodrigues M, Romaldini C. I Inquérito Nacional Brasileiro sobre Doença Celíaca. SPGNP Boletim Informativo 1993;I(2):6-8.

4. Barbieri D. Doença celíaca. In: Barbieri D, Koda YKL. Doenças gastroenterológicas em pediatria. São Paulo: Atheneu; 1996. p.176-88.

5. Bottaro G, Failla P, Rotolo N, Santifilippo G, Azzaro F, Spina M, Patane R. Changes in coeliac disease behavior over the years. Acta Paediatr 1993;82:566-8.
6. Bottaro G, Cataldo F, Rotolo N, Spina M, Corazza GR. The clinical pattern of subclinical/silent celiac disease: an analysis on 1026 consecutive cases. Am J Gastroenterol 1999;94:691-6.

7. Ceccarelli M, Caiulo VA, Cortigiani L, Pucci C, Lupetti L, Ughi C. Gli aspetti clinici della malattia celiaca. Confronto tra due periodi: prima e dopo l'introduzione del dosaggio degli anticorpi antigliadina nella pratica clinica. Minerva Pediatr 1990;42:263-6.

8. Ciclitira PJ. American Gastroenterological Association review on celiac sprue Gastroenterology 2001;120:1526-40.

9. Fleiss JL. Statistical methods for rates and proportions. $2^{\text {nd }}$ edition. New York: John Wiley; 1981.

10. Galvão LC, Gomes RC, Ramos AMO. Doença celíaca: relato de 20 casos no Rio Grande do Norte. Arq Gastroenterol 1992;29:28-33.

11. Gandolfi L, Pratesi R, Cordoba JCM, Tauil PL, Gasparin M, Catarsi C. Prevalence of celiac disease among blood donors in Brazil. Am J Gastroenterol 2000;95:689-92. 
12. Godkin A, Jewell D. The pathogenesis of celiac disease. Gastroenterology 1998;115:206-10.

13. Gomez F. Desnutrition. Bol Med Hosp Infant Mex 1946;3:543.

14. Hansson T, Dalbom I, Rogberg S, Dannaeus A, Hopfl P, Gut H, Klarescog L. Recombinant human tissue transglutaminase for diagnosis and follow-up of childhood coeliac disease. Paediat Res 2002;51:700-5.

15. Hollander M, Wolfe DA. Nonparametric statistical methods. New York: John Wiley; 1973

16. Koda YK, Barbieri D. Doença celíaca. Estudo clínico em 27 crianças: problemas no retardo diagnóstico. Pediatria (São Paulo) 1983;5:38-41.

17. Maki M, Kallonen K, Lahdeaho ML, Visakorpi JK. Changing pattern of childhood coeliaca disease in Finland. Acta Paediatr Scand 1988;77:408-12.

18. Melo SBC. Prevalência de doença celíaca em doadores de sangue em Ribeirão Preto [dissertação]. Ribeirão Preto: Faculdade de Medicina de Ribeirão Preto da Universidade de São Paulo; 2003.

19. Meuwisse GW. Diagnostic criteria in coeliac disease. Acta Paediatr Scand 1970;59:461-3

20. Murray JA. The widening spectrum of celiac disease. Am J Clin Nutr 1999;60:354-65.

21. National Center for Health Statistics (NCHS). Growth curves for children. Washington, DC: US Department of Health Education Welfare Public-Health Service; 1977. (DHEW publication $\mathrm{N}^{\circ}$ (PHS) 78) p.1650.

22. Penna FJ, Carvalho AST, Roquete MLV, Mota JAC, Duarte MA, Torres MR, PeretFilho LA, Figueiredo-Filho PP, Starling AL. Doença celíaca. Aspectos atuais e nossa experiência. J Pediatr (Rio de J) 1984;57:325-9.
23. Sanders DS, Patel D, Stephenson TJ, Ward AM, McCloskey EV, Hadjivassiliou M, Lobo AJ. A primary care cross-sectional study of undiagnosed adult coeliac disease. Eur J Gastroenterol Hepatol 2003;15:407-13.

24. Schuppan D. Current concepts of celiac disease pathogenesis. Gastroenterology 2000;119:234-42

25. Sdepanian VL, Fagundes-Neto U, Morais MB. Doença celíaca: características clínicas e métodos utilizados no diagnóstico de pacientes cadastrados na Associação dos Celíacos do Brasil. J Pediatr (Rio de J) 2001;77:131-8.

26. Silva EMBT, Fernandes MIM, Galvão LC, Sawamura R, Donadi EA. Human leukocyte antigen class II alleles in white Brazilian patients with celiac disease. J Pediatr Gastroenterol Nutr 2000;31:391-4.

27. Troncone R, Greco L, Auricchio S. Gluten-sensitive enteropathy. Pediatr Clin North Am 1996;43:355-73.

28. Visakorpi JK, Maki M. Changing clinical feature of coeliac disease. Acta Paediatr 1994; (suppl 395):10-3.

29. Walker-Smith JA, Guandalini S, Schmitz J, Shmerling DH, Visakorpi JK. Revised criteria for diagnosis of coeliac disease. Arch Dis Child 1990;65:909-11.

30. Waterloo JC. Classification and definition of protein-calorie malnutrition. Br Med J 1972;2:566-9. 